\title{
Sex-related differences regarding cephalic vein lead access for CIEDs implantation
}

\author{
Jane Taleski ${ }^{1^{*} \mathbb{D}}$, Svetlana Stankovik ${ }^{2}$, Dejan Risteski ${ }^{1}$, Filip Janusevski ${ }^{1}$, Bekim Pocesta ${ }^{1}$, Zan Zimbakov ${ }^{1}$ and \\ Lidija Poposka'
}

\begin{abstract}
Background: Minimally invasive and safe central venous access is imperative for lead insertion of cardiac implantable electronic devices (CIEDs). The purpose of this trial was to explore and compare the usability of the cephalic vein (CV) between both sexes.

Methods and results: This single-center prospective study included 102 consecutive patients in a period of six months. Pre-procedural contrast-enhanced venographic images of the upper arm were performed in all included patients. Our attention was focused on comparing several morpho-anatomical CV characteristics such as venous diameter, presence of valves and angle of entrance of the CV into the subclavian vein (SV). Study results concerning the CV morpho-anatomical differences were more favorable regarding the female patient group, with significant differences in $\mathrm{CV}$ diameter ( $p$-0.030). There was also a difference in favor of the female group regarding the favorable CV angle of entrance into the SV, found in the $61.7 \%$ versus $54.4 \%$ in the male patient group. The comparison of usability of the CV and CVC technique was explored by comparing the number of leads inserted through the CV in both sexes. Two leads were implanted in $11.7 \%$ in the female group versus $5.8 \%$ in the male group, and 0 leads through the CV in $38.2 \%$ of the female patients versus $50 \%$ of male group.
\end{abstract}

Conclusion: Female patients have more favorable cephalic vein morpho-anatomical futures and better usability for lead placement than male patients.

Keywords: Sex differences, Cephalic vein, Axillary vein, Subclavian vein, Device implantation

\section{Background}

The number of cardiac implantable electronic devices (CIED) implantation procedures has been increasing exponentially in the past few decades [1].

Central venous access that is minimally invasive and safe is imperative for lead insertion of cardiac implantable electronic devices (CIEDs) such as permanent pacemakers, implantable cardioverter defibrillators, and cardiac resynchronization therapy [2]. Numerous reports

\footnotetext{
*Correspondence: jtaleski@gmail.com

${ }^{1}$ Department of Cardiac Electrophysiology and Electrostimulation,

University Clinic of Cardiology, Bul. Mother Teresa 17,

1000 Skopje, Republic of North Macedonia

Full list of author information is available at the end of the article
}

have explained modification of CIED implantation techniques [3-5]. Needless to say that no account can totally cover all the implant techniques and their variations used by different operators. As any practical skill, written descriptions cannot replace the practical experience of a skilled implanter [3].

CIED implantation technique can be divided in several stages. The first stage begins by gaining central venous access for lead insertion. This fundamental step can be broadly divided into techniques involving direct visualization of the target vein by a cut-down technique (most commonly the cephalic vein), or those involving needle puncture of the vein [6]. Cephalic vein cut-down $(\mathrm{CVC})$ followed by subclavian vein puncture (SVP) and recently added fluoroscopy-guided axillary 
vein puncture (AVP) techniques are widely used for lead insertion in clinical practice. However, the use of one technique over the other is largely limited by operator experience and local working strategies $[7,8]$.

Subclavian vein access was associated with increased rates of early and late complications. On the other hand, CVC technique requires longer learning curve and can have lower success rates. Numerous operators consider AVP technique to be suitable alternative in terms of safety and efficacy [8]. The subclavian/ axillary vein approach can be used as a first or alternative choice, especially because of a more rapid learning curve. Less-experienced implanters are usually more confident with SVP technique, particularly if an extrathoracic approach is used [9]. In both techniques, it is highly recommended to have fluoroscopic guidance especially for axillary and intrathoracic subclavian vein puncture [5]. These approaches can result in complications like pneumothorax, hemopneumothorax, inadvertent subclavian artery puncture, brachial nerve plexus injury, thoracic duct injury and mechanical damage to the lead(s) [10].

Many centers, especially academic, prefer to use the cephalic vein cut-down (CVC) technique as their first choice. The cephalic vein's usual location is in deltopectoral groove, penetrating the clavipectoral fascia to join the axillary vein medial to the pectoralis minor muscle and together continuing as the subclavian vein [2]. Naturally, there can be different variations and anomalies of the cephalic vein and the tissues around it. These variations and anomalies may prolong the procedure time, and result in unsuccessful cephalic access in $40 \%$ of cases, especially pronounced in less-experienced operators. Improvements of this technique have been described, like the use of a hydrophilic guidewire when direct lead insertion failed and cannulation of retro-pectoral veins in cases of insufficient caliper of the cephalic vein. Also, two additional techniques were introduced, namely the simultaneous use of two guidewires and the use of stiff angiography guidewires. These techniques can reduce failure rates up to $10-20 \%[11,12]$.

The purpose of this study was to explore and compare the usability of the cephalic vein $(\mathrm{CV})$ between both sexes. First by comparing several morpho-anatomical parameters, like CV diameter, the presence of valves and the angle of entry into the SV and secondly by determining the number of leads placed through the $\mathrm{CV}$ or through an alternative venous access site (SVP/ AVP techniques). With this trial, we tried to answer the question of sex-related differences in cephalic vein usability as a central venous access site for pacing leads.

\section{Methods}

This single-center prospective study included 102 consecutive patients referred for CIED implantation in the period between March and September 2015.

Pre-procedural contrast-enhanced venographic images (in PA, posterior-anterior projection) of the upper arm were made in all included patients, and they were interpreted directly after each procedure. Our attention was focused on comparing several morphoanatomical $\mathrm{CV}$ characteristics such as venous diameter, presence of valves and angle of entrance of the $\mathrm{CV}$ into the SV.

\section{Procedure}

All CIED implantation procedures were made in a sterile electrophysiology laboratory. Anticoagulated patients with low thromboembolic risk were operated after suspension of anticoagulant treatment to minimize bleeding risk. Those with moderate-to-high thromboembolic risk were operated under anticoagulant treatment, avoiding bridge therapy with heparin. Antibiotic prophylaxis was administered to all patients. Procedures were performed from both left and right side under local anesthesia (Lidocaine injection bp 2\%). Once the cutaneous incision was created and the subcutaneous tissue dissected, the appointed venous access site was used. Afterward, CIED implantation procedures were identical in all included patients and were performed in the standard way. The preferred and first choice for central venous access was cephalic vein cut-down technique (CVC) for one or two leads. In those cases where we were unable to do so, we tried advancing with hydrophilic wire before switching to subclavian/axillary vein puncture access techniques (SVP/AVP), guided by pre-procedural venographic images. As an alternative, if needed we performed extrathoracic fluoroscopic-guided SV puncture or AV puncture guided by contrast venography.

The study included implantation of single/dualchamber permanent pacemakers (PPM), dual-chamber implantable cardioverter defibrillator (ICD) and one cardiac resynchronization therapy device (CRT-p). All procedures were performed by experienced operators $(>100$ CIED per year and center complication rate $1.8 \%$ per year).

\section{Pre-procedural venography}

Images were made before the application of local anesthesia, with $10 \mathrm{ml}$ of undiluted contrast (Ultravist 370Bayer AG, Leverkusen, Germany) which was injected, through the left/right cubital vein. The pre-procedural venography images gave a clear morpho-anatomical 
picture of the $\mathrm{CV}, \mathrm{AV} / \mathrm{SV}$ and their mutual connections, just before the beginning of the implantation procedure.

At the end of every procedure, we stored fluoroscopic images of the deltopectoral groove to assess the position of the pacing generator and pacing leads.

\section{Definitions}

Cephalic vein diameter-After the procedure, we used DSA (digital subtraction angiography) to perform measurements of the diameter of the cephalic vein used for implantation of the leads. The diameter was measured at the narrowest part of the vein, parallel to the head and neck of the humerus (Fig. 1). Cases with CV constriction were measured on the narrowest measurable part of the vein.

Angle of entrance-This variable represents angle of entrance of the CV into the SV. We considered an angle between 0 and 90 degrees pointing medially as favorable for CV usage and lead placement [Fig. 2].

Valves-We counted the number of valves in the part of $\mathrm{CV}$ that we use for lead placement, and in this trial results, 1 or less valves in both sexes were considered as favorable [Fig. 3].

Procedural time-In all included patients, we measured the time of the first stage of every procedure, starting from injecting the local anesthesia until we introduced the leads, regardless of which vein we use to obtain central venous access.

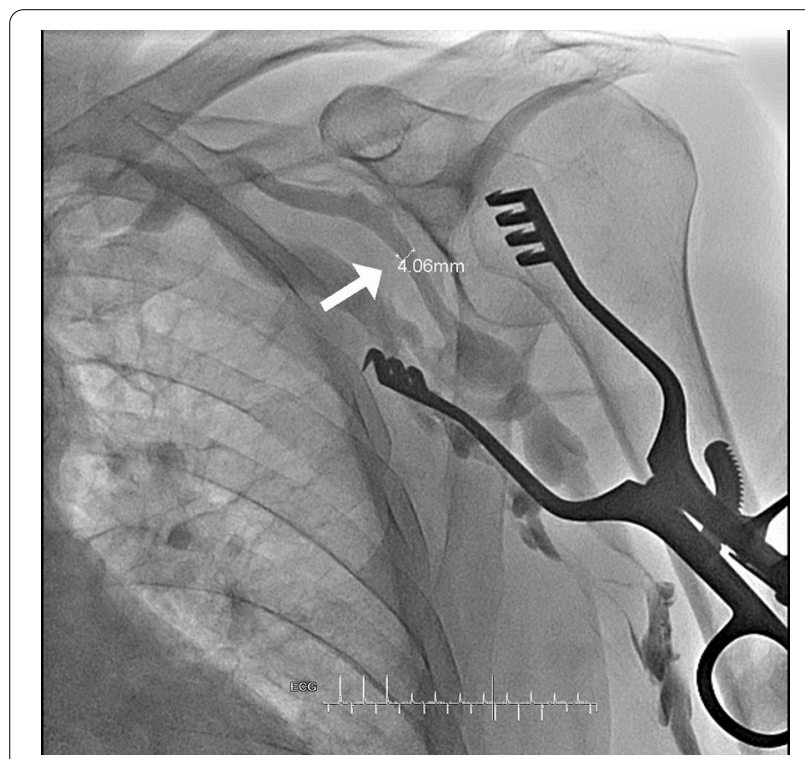

Fig. 1 Measurement of the Cephalic vein diameter using digital subtraction angiography DSA (white arrow)

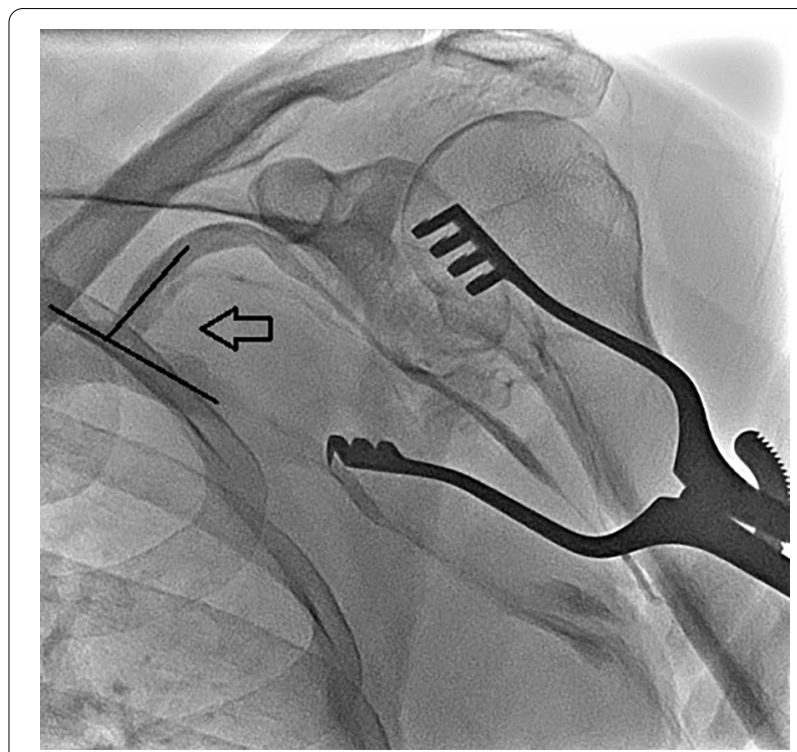

Fig. 2 Black arrow is pointing to the angle of entrance of the cephalic vein into the subclavian vein, drawn with black lines

\section{Statistical analysis}

Categorical variables are expressed as numbers and percentages and continuous variables are expressed as the mean \pm standard deviation or median (interquartile range). To assess the role of sex, age, $\mathrm{CV}$ diameter, angle of entrance, and number of valves $\leq 1$, hierarchical multiple regression model was tested for number of leads through CV. Sex and age were entered in the first step, while CV diameter, angle of entrance, and number

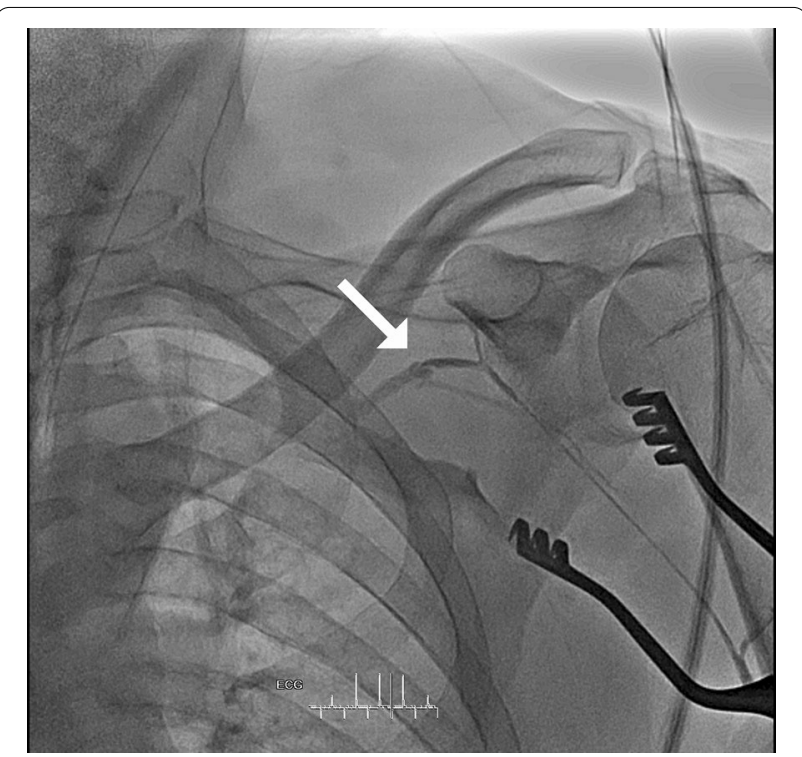

Fig. 3 Presence of valves into the cephalic vein (white arrow) 
of valves $\leq 1$ were entered in the second step. Statistical analysis was performed using the Statistical Package for Social Sciences software version 17.0 for Windows (IBM Corp., Armonk, NY, USA).

\section{Results}

During a period of one year, we evaluated (102) venography-guided CIED implantation procedures. Sixtyeight $(66.6 \%)$ patients were male and $34(33.3 \%)$ were female. The mean age of the female group of patients was 73.9 years old, and in male group 70.5 years old $(\mathrm{p}<0.0001)$. Ninety-eight $(96 \%)$ CIED were implanted from the left side and only four (3.9\%) of CIED from the right (deltopectoral) side.

During this period of time 98 (96\%) of the CIEDs implanted were permanent pacemakers (PPM). This group included 41 dual-chamber pacemakers (DDD), seven single-chamber pacemakers with atrial sensing coil (VDD) and 48 single-chamber pacemakers (VVI). This trial also included three (2.9\%) dual-chamber implantable cardioverter defibrillators (ICD) and one (0.9\%) cardiac resynchronization therapy device (CRT). Ninety-seven (95\%) of all implanted pacing/defibrillation leads were with active fixation and only five $(4.9 \%)$ with passive fixation.

In the majority of patients, 55 (53.9\%), CV was used as central venous access site for pacing leads, and in 46 (45\%), at least one lead was introduced by using CVC technique. Regarding the sex-related differences in the utilization CVC technique and the number of implanted leads through this venous access, we obtained the following results.

We can see the main difference in usability of the CV in both sexes regarding the number of implanted leads, especially with the fact that two leads were implanted in $11.7 \%$ in the female group versus $5.8 \%$ in the male group, and zero leads through the $\mathrm{CV}$ in $38.2 \%$ of the female patient's versus $50 \%$ of male.

One of the main goals of this trial was to explore the morpho-anatomical differences of the $\mathrm{CV}$ in both sexes. Several variables were explored and compared. We found a significant difference $(p-0.030)$ regarding the $\mathrm{CV}$ diameter. The mean diameter value in the female group was 3.5 versus 2.9 in the male group. There was also a difference in favor of the female group regarding the $\mathrm{CV}$ angle of entrance into the SV. Favorable angle of entrance was found in the $61.7 \%$ of the female patients versus $54.4 \%$ of the male patients. Regarding the last variable, there were some differences in favor of the male group of patients. A total of $34.3 \%$ of the female patients had one or less valves in the $\mathrm{CV}$ versus $50 \%$ in the male group (Tables 1 and 2).

It is also important to note that factors that could negatively influence vein diameter, such as CKD and diabetes
Table 1 Patient characteristics

\begin{tabular}{|c|c|c|}
\hline Variable & Female $(n=34)$ & Male $(n=68)$ \\
\hline Age (years) & $73.9(35-85)$ & $70.5(56-86)$ \\
\hline $\mathrm{BMI}\left(\mathrm{kg} / \mathrm{m}^{2}\right)$ & $27(17-41)$ & $30(17-37)$ \\
\hline Hypertension & $29(85 \%)$ & $60(88 \%)$ \\
\hline Diabetes & $11(32 \%)$ & $12(17 \%)$ \\
\hline$C K D^{a}$ & $1(2.9 \%)$ & $1(1.4 \%)$ \\
\hline$C A D^{b}$ & $4(11.7 \%)$ & $17(25 \%)$ \\
\hline \multicolumn{3}{|l|}{ CIED ${ }^{c}$ indication } \\
\hline AV block gr III & $17(16.6 \%)$ & $19(18.6 \%)$ \\
\hline Mobitz II & $10(9.8 \%) 19(18.6 \%)$ & 19 (18.6\%) \\
\hline Mobitz I & 0 & $1(0.9 \%)$ \\
\hline AFib $^{d}$ & $4(3,9 \%)$ & $13(12.7 \%)$ \\
\hline SSSy ${ }^{\mathrm{e}}$ & $2(1.9 \%)$ & $9(8.8 \%)$ \\
\hline$V T^{f}$ & 0 & $3(2.9 \%)$ \\
\hline $\mathrm{PM}^{\mathrm{g}}$ upgrade to $\mathrm{CRT}^{\mathrm{h}}$ & 0 & $1(0.9 \%)$ \\
\hline
\end{tabular}

${ }^{\mathrm{a}}$ Chronic kidney disease

${ }^{\mathrm{b}}$ Coronary artery disease

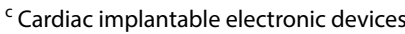

${ }^{\mathrm{d}}$ Atrial fibrillation

e Sick sinus syndrome

${ }^{f}$ Ventricular tachycardia

${ }^{g}$ Pacemaker

${ }^{\mathrm{h}}$ Cardiac resynchronization therapy

Table 2 Anatomical and functional cephalic vein (CV) differences between sexes

\begin{tabular}{|c|c|c|c|}
\hline Variable & Female & Male & $P<0.03$ \\
\hline \multicolumn{4}{|c|}{ Number of leads through CV } \\
\hline 0 leads & $38 \%$ & $50 \%$ & NS \\
\hline 1 lead & $50 \%$ & $44.1 \%$ & NS \\
\hline 2 leads & $11.7 \%$ & $5.8 \%$ & NS \\
\hline CV diameter & 3.5 & 2.9 & 0.030 \\
\hline Angle of entrance & $61.7 \%$ & $54.4 \%$ & 0.054 \\
\hline Valves $\leq 1$ & $34.3 \%$ & $50 \%$ & 0.531 \\
\hline
\end{tabular}

were more prevalent in the female group of patients with $2.9 \%$ versus $1.4 \%$ and $32 \%$ versus $17 \%$ accordingly.

Regarding the procedural complication rates, there were no significant differences in both sexes. Procedural time for both groups, where CVC technique was used, was between $8 \pm 1 \mathrm{~min}$, and for SVP/AVP techniques $6 \pm 2 \mathrm{~min}$. However, in some patients, we were forced to switch to SVP/AVP techniques due to long procedural time (>20 min) spent on CVC technique.

Standardized beta coefficients for the independent predictors of number of leads through $\mathrm{CV}$ in the study sample are shown in Table 3. Angle of entrance $(\beta=0.61$, 
Table 3 Hierarchical multiple regression model for number of leads through CV in the study sample

\begin{tabular}{|c|c|c|c|c|c|c|c|}
\hline \multicolumn{2}{|c|}{ Number of leads through CV } & \multirow[t]{2}{*}{ B } & \multirow[t]{2}{*}{ SE } & \multicolumn{2}{|c|}{$95 \% \mathrm{Cl}$ for $\mathrm{B}$} & \multirow[t]{2}{*}{ Beta } & \multirow[t]{2}{*}{$R^{2}$} \\
\hline & & & & Lower & Upper & & \\
\hline \multirow[t]{3}{*}{ Step 1} & Male sex & -0.07 & 0.15 & -0.37 & 0.23 & -.05 & 0.006 \\
\hline & Age & -0.004 & 0.01 & -0.02 & 0.01 & -.06 & \\
\hline & Constant & 1.08 & 0.57 & -0.05 & 2.2 & & \\
\hline \multirow[t]{6}{*}{ Step 2} & Male sex & -0.16 & 0.11 & -0.39 & 0.07 & -.13 & 0.482 \\
\hline & Age & -0.004 & 0.01 & -0.02 & 0.01 & -.06 & \\
\hline & CV diameter & 0.04 & 0.04 & -0.04 & 0.11 & .09 & \\
\hline & Angle of entrance & 0.83 & 0.12 & 0.6 & 1.06 & $.61^{* *}$ & \\
\hline & Number of valves $\leq 1$ & 0.34 & 0.11 & 0.12 & 0.55 & $.27^{*}$ & \\
\hline & Constant & 0.25 & 0.44 & -0.63 & 1.12 & & \\
\hline
\end{tabular}

$R 2=.006$ for Step $1 ; \Delta R 2=.476$ for Step $2(P<0.001){ }^{*} p<0.01,{ }^{* *} p<0.001$

$p<0.001)$ and number of valves $\leq 1 \quad(\beta=0.27, p<0.01)$ positively predicted number of leads through CV. Preferable angle of entrance and low number of valves (valves $\leq 1$ ) were associated with a higher number of leads through CV. Transition from male to female patient group also predicted higher number of leads through $\mathrm{CV}$, but this association $(\beta=-0.13, p>0.05)$ was not significant.

\section{Discussion}

Study results showed an important difference regarding the CV morpho-anatomical characteristics and CVC technique usability, between sexes. There was a significant difference in $\mathrm{CV}$ diameter in favor of the female patient group, also there was difference in favor of the same group regarding the angle of entrance as well. Regarding the usability of the $\mathrm{CV}$ and $\mathrm{CVC}$ technique, we explored the number of implanted leads, and again there was difference in favor of the female patient group. Clearly, there is a directly proportional relation between morpho-anatomical characteristics of the $\mathrm{CV}$ and the CVC technique usability, where this trial showed an advantage of the female patient group. These results were confirmed with multivariable analysis, which showed that $\mathrm{CV}$ usability increases as we move from male to female patients. It also showed that $\mathrm{CV}$ angle of entrance and low or no valves are significant factors for increased $\mathrm{CV}$ usability. Our high failure rate shows that the CVC technique is technically more complex approach, and it needs a longer learning curve to reach higher success rate $[2,4]$. However, as study results showed, learning curve for CVC technique is not the only parameter that influences its success, sex-related morpho-anatomical differences can be crucial for successful lead passage also.

Cephalic vein cut-down $(\mathrm{CVC})$, subclavian vein puncture (SVP) and axillary vein puncture (AVP) are the three most commonly used techniques for transvenous access. CVC was found to be the venous access of choice in $60 \%$ of the centers in Europe in a survey [13]. This technique has its advantages and disadvantages over SV/AV puncture techniques $[4,14]$. In a meta-analysis, CVC technique was associated with a lower risk of pneumothorax and lead failure compared with SVP; however, there was no significant difference in the risk of pocket hematoma/ bleeding or device infection with CVC compared with SVP. The same meta-analysis showed that the acute procedural success was higher with AVP and SVP compared with $\mathrm{CVC}$, and total procedure time was significantly longer with CVC compared with AVP but not SVP [2].

Successful lead passage through the CV or CVC technique usability depends on the morpho-anatomical parameters of the vessel and other factors like size and structure of the leads introduced via the $\mathrm{CV}$, vessel wall elasticity and operator's experience [1, 10, 15]. Morphoanatomical characteristics of the CV were analyzed and associated with successful lead passage in one prior clinical trial. In that same trial, contrast venography results, obtained during first-time lead placement were analyzed. Venography was indicated only in cases of problematic lead introduction (15\%) with either the CVC or AVP/SVP techniques. This clinical trial results showed that most common unfavorable morpho-anatomical parameters were CV diameter of $\leq 1 \mathrm{~mm}$ (18\%), sharp curvature of the terminal $\mathrm{CV}$ segment as it joined the axillary vein (14\%) and terminal CV bifurcation (9\%) [1].

In one study, peri-procedural scoring system was presented for morpho-anatomical evaluation of the cephalic vein, which should facilitate the choice between the CVC and SVP/AVP techniques. Results from this trial were very encouraging regarding peri-procedural score safety and efficacy and should allow less-experienced implanters to gain more confidence during the procedure [16]. 
Another prospective study showed $17 \%$ of failure rate in lead placement through CV approach, associated with the small vessel diameter [15]. In addition to this morpho-anatomical parameter of the $\mathrm{CV}$, a different study described a technique where pre-procedural ultrasonography was used for the vessel assessment, to minimize the failure rate [17]. Various studies have described alterations to the classical CV cut-down technique, including the use of a hydrophilic guidewire, cannulation of retropectoral veins, simultaneous use of two guidewires and the use of stiff angiography guidewires. These so-called "no puncture" techniques were successful in over $95 \%$ of implantations, without any major complications $[11,12$, 18].

Most common short-term complications related with central venous access and lead insertions are pneumothorax, lead dislodgement, and pocket hematoma/bleeding, reportedly range from 8 to $12 \%[19,20]$. In a Danish pacemaker register, Kirkfeldt et al. reported that the risk of pneumothorax was almost 8 times higher with SVP in contrast with CVC technique [21]. In addition, SVP is known to be linked with higher risk of lead failure and fractures due to the phenomenon of "subclavian crush," where the lead is mechanically ensnared between the costo-clavicular ligament and subclavius muscle [22]. One clinical trial reported that lead failures occurred mainly with ICD leads, a lesser age, the puncture method, lead model, and diagnosis of idiopathic ventricular fibrillation and Brugada syndrome [23].

However, CVC is the safest venous access obtainable for lead insertion. This access site avoids all the complications linked to SVP such as pneumothorax and subclavian crush syndrome and all at a reduced cost. Nevertheless, because it is a cut-down technique, CVC requires surgical experience with a steep learning curve, often associated with lower success rate and higher blood loss $[9,10]$. In addition, AVP remains a suitable alternative with similar safety and efficacy as CVC technique, with shorter learning curve. In the same meta-analysis, they confirmed the need for continuous widespread dispersion and adoption of CVC technique [2].

In this retrospective study, we compared several important morpho-anatomical features of the $\mathrm{CV}$ and CVC technique in male and female patients scheduled for CIED implantation procedure, and at the same time, we demonstrated the advantage of this approach in the female population of patients.

\section{Limitations of the study}

This was a retrospective single-center study with limited number of patients. More prospective, randomized multi-center studies are needed to explore our results.

\section{Conclusion}

Cephalic vein should be the first choice for CIED lead(s) implantation. Female patients have more favorable cephalic vein morpho-anatomical features and better usability for lead placement than male patients. Understanding these CV features will lead to its more efficient and safer utilization.

\begin{abstract}
Abbreviations
CIED: Cardiac implantable electronic devices; CVC: Cephalic vein cut-down technique.; AVP: Axillary vein puncture; SVP: Subclavian vein puncture; CV: Cephalic vein; SV: Subclavian vein; PPM: Permanent pacemaker; ICD: Implantable cardioverter defibrillator; CRT: Cardiac resynchronization therapy; DSA: Digital subtraction angiography; CKD: Chronic kidney disease; CAD: Coronary artery disease; AFib: Atrial fibrillation;VT: Ventricular tachycardia; PM: Pacemaker; SSSy: Sick sinus syndrome.
\end{abstract}

\section{Authors' contributions}

JT analyzed and interpreted the patient data and a major contributor in writing the manuscript. LP interpreted the data and contributed in writing the manuscript. DR, FJ and BP analyzed the patient data. SS and ZZ supervised the interpretation of the data and the manuscript. All authors read and approved the final manuscript.

\section{Funding}

Not applicable.

\section{Availability of supporting data}

The datasets used and/or analyzed during the current study are available from the corresponding author on reasonable request.

\section{Declarations}

Ethical approval and consent to participate

The study protocol adhered to the Declaration of Helsinki and was approved by the institutional review board.

\section{Consent for publication}

Not applicable.

\section{Competing interests}

The authors declare that they have no competing interests.

\section{Author details}

'Department of Cardiac Electrophysiology and Electrostimulation, University Clinic of Cardiology, Bul. Mother Teresa 17, 1000 Skopje, Republic of North Macedonia. ${ }^{2}$ University Clinic of Hematology, 1000 Skopje, Republic of North Macedonia.

Received: 10 June 2021 Accepted: 12 September 2021

Published online: 17 November 2021

\section{References}

1. Steckiewicz R, Świętoń E, Stolarz P, Grabowski M. Clinical implications of cephalic vein morphometry in routine cardiac implantable electronic device insertion. Folia Morphol (Warsz). 2015;74:458-64.

2. Atti V, Turagam MK, Garg J, Koerber S, Angirekula A, Gopinathannair R, et al. Subclavian and axillary vein access versus cephalic vein cutdown for cardiac implantable electronic device implantation: a meta-analysis. JACC Clin Electrophysiol. 2020;6:661-71.

3. Rajappan K. Permanent pacemaker implantation technique: part II. Heart. 2009:95:334-42. 
4. Parsonnet $\mathrm{V}$, Roelke $\mathrm{M}$. The cephalic vein cutdown versus subclavian puncture for pacemaker/ICD lead implantation. Pacing Clin Electrophysiol. 1999;22:695-7.

5. Sharma G, Senguttuvan NB, Thachil A, Leong D, Naik N, Yadav R, et al. A comparison of lead placement through the subclavian vein technique with fluoroscopy-guided axillary vein technique for permanent pacemaker insertion. Can J Cardiol. 2012;28:542-6.

6. Lau EW. Upper body venous access for transvenous lead placementreview of existent techniques. Pacing Clin Electrophysiol. 2007;30:901-9.

7. Chan NY, Kwong NP, Cheong AP. Venous access and long-term pacemaker lead failure: comparing contrast-guided axillary vein puncture with subclavian puncture and cephalic cutdown. Europace. 2017;19:1193-7.

8. Jiménez-Díaz J, Hiquera-Sobrino F, Piqueras-Flores J, Pérez-Díaz P, González-Marín MA. Fluoroscopy-guided axillary vein access vs cephalic vein access in pacemaker and defibrillator implantation: Randomized clinical trial of efficacy and safety. J Cardiovasc Electrophysiol. 2019;30:1588-93.

9. Calkins H, Ramza BM, Brinker J, Atiga W, Donahue K, Nsah E, et al. Prospective randomized comparison of the safety and effectiveness of placement of endocardial pacemaker and defibrillator leads using the extrathoracic subclavian vein guided by contrast venography versus the cephalic approach. Pacing Clin Electrophysiol. 2001;24:456-64.

10. Tobin K, Stewart J, Westveer D, Frumin H. Acute complications of permanent pacemaker implantation: their financial implication and relation to volume and operator experience. Am J Cardiol. 2000;85:774-6.

11. Neri R, Cesario AS, Baragli D, Monti F, Danisi N, Glaciale G, et al. Permanent pacing lead insertion through the cephalic vein using a hydrophilic guidewire. Pacing Clin Electrophysiol. 2003;26:2313-4.

12. Kolettis TM, Lysitsas DN, Apostolidis D, Baltogiannis GG, Sourla E, Michalis LK. Improved "cut-down" technique for transvenous pacemaker lead implantation. Europace. 2010;12:1282-5.

13. Bongiorni MG, Proclemer A, Dobreanu D, Marinskis G, Pison L, BlomstromLundqvist C; Scientific Initiative Committee, European Heart Rhythm Association. Preferred tools and techniques for implantation of cardiac electronic devices in Europe: results of the European Heart Rhythm Association survey. Europace. 2013;15:1664-8.

14. Ussen B, Dhillon PS, Anderson L, Beeton I, Hickman M, Gallagher MM. Safety and feasibility of cephalic venous access for cardiac resynchronization device implantation. Pacing Clin Electrophysiol. 2011;34:365-9.

15. Knight BP, Curlett K, Oral H, Pelosi F, Morady F, Strickberger SA. Clinical predictors of successful cephalic vein access for implantation of endocardial leads. J Interv Card Electrophysiol. 2002;7:177-80.

16. Taleski J, Poposka L, Janusevski F, Pocesta B, Boskov V, Boyle NG. Scoring system assessment of cephalic vein access for device implantation. J Innov Card Rhythm Manag. 2018;9:3284-90.

17. Chen JY, Chang KC, Lin KH, Lin YC, Lee JD, Huang SK. Ultrasonographic predictors of unsuccessful cephalic vein approach during pacemaker or defibrillator lead implantation. Pacing Clin Electrophysiol. 2006;29:706-11.

18. Camous JP, Raybaud F, Lesto I, Benoit PH. Introduction of permanent cardiac stimulation/defibrillation leads via the retro-pectoral veins. Pacing Clin Electrophysiol. 2005;28:324-5.

19. Kirkfeldt RE, Johansen JB, Nohr EA, Jørgensen OD, Nielsen JC. Complications after cardiac implantable electronic device implantations: an analysis of a complete, nationwide cohort in Denmark. Eur Heart J. 2014;35:1186-94.

20. Udo EO, Zuithoff NP, van Hemel NM, de Cock CC, Hendriks T, Doevendans $\mathrm{PA}$, et al. Incidence and predictors of short- and long-term complications in pacemaker therapy: the FOLLOWPACE study. Heart Rhythm. 2012;9:728-35

21. Kirkfeldt RE, Johansen JB, Nohr EA, Moller M, Arnsbo P, Nielsen JC. Pneumothorax in cardiac pacing: a population-based cohort study of 28,860 Danish patients. Europace. 2012;14:1132-8.

22. Magney JE, Flynn DM, Parsons JA, Staplin DH, Chin-Purcell MV, Milstein S, et al. Anatomical mechanisms explaining damage to pacemaker leads, defibrillator leads, and failure of central venous catheters adjacent to the sternoclavicular joint. Pacing Clin Electrophysiol. 1993;16:445-57.

23. Aizawa Y, Negishi M, Kashimura S, Nakajima K, Kunitomi A, Katsumata Y, et al. Predictive factors of lead failure in patients implanted with cardiac devices. Int J Cardiol. 2015;199:277-81.

\section{Publisher's Note}

Springer Nature remains neutral with regard to jurisdictional claims in published maps and institutional affiliations.
Ready to submit your research? Choose BMC and benefit from:

- fast, convenient online submission

- thorough peer review by experienced researchers in your field

- rapid publication on acceptance

- support for research data, including large and complex data types

- gold Open Access which fosters wider collaboration and increased citations

- maximum visibility for your research: over $100 \mathrm{M}$ website views per year

At BMC, research is always in progress.

Learn more biomedcentral.com/submissions 